\title{
Nature Immunology turns 20
}

\author{
Twenty years ago, a new immunology journal was launched. The editors now celebrate the anniversary of Nature \\ Immunology by looking at the past, present and future.
}

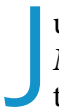
uly 2020 marks the 20th anniversary of Nature Immunology's launch. As we take time to reflect on the successes of the last 20 years, we do so acknowledging that our progress is in stark contrast to the context of 2020. Our world has been altered by the harsh reality of the COVID-19 pandemic and the protests against institutionalized racism and discrimination. Nature Immunology, along with the rest of Springer Nature, seeks to be a part of the solution in both of these fights.

Nature Immunology was launched by the chief editor, Linda J. Miller, and two associate editors, Jamie D. K. Wilson and Melanie Brazil. At the time, there were two key premier journals serving the immunology community - the Journal of Experimental Medicine and Immunity, both run by editorial boards, although Immunity has now changed this model. Like other Nature research journals, Nature Immunology avoided using an editorial board. As Linda put it, “...immunology was [also] on the cusp of a rise in popularity, given the growing interest in innate immunity, $\mathrm{T}$ cell education, and regulatory mechanisms beyond cell death, such as the re-emergence of good studies on $\mathrm{T}$ cells that suppressed the activity of other T cells. Progress was being made in asthma and inflammation, how cancers evaded the immune system, TLRs, and mucosal immunity", to name but a few areas of interest. The time was ripe for another broad immunology journal.

Thus, a new journal was born, with the mission of providing a platform for cutting edge research that yields fundamental insight into the workings of the immune system, covering all areas of immunology. The journal soon became one of the highest ranking titles for the immunology community (according to Clarivate). Part of this success stems from our editorial team, which has fortunately remained fairly stable over the last 20 years. In 2001, Laurie Dempsey joined the team and is now a veteran editor well known in the community. Following Melanie's departure, we were joined by Peter Lee, Douglas Braaten and Christine Borowski during our first decade, all of whom later forged successful careers post-Nature Immunology. Linda passed the baton to Jamie in 2005. Thereafter, Ioana Visan and Zoltan Fehervari joined in 2009 and 2010, respectively. Collectively, the current team has over 60 years' editorial experience!

Of course, much has changed in the publishing landscape since 2000. Long gone are the times when we received manuscripts by mail and edited using pen and paper! But Nature Immunology, and indeed Springer Nature as a whole, has taken great pride in our innovations and in meeting author needs over the years. Of particular note, Nature-branded journals have driven an increase in transparency and open science. Before sending a paper to peer review, we mandate that authors supply a reporting checklist, which is intended to improve the accuracy of experimental reporting and to aid each referee's assessment of the data. Alongside this, authors of manuscripts that contain large datasets are required to deposit them in public repositories and to make them accessible to reviewers. Access to all raw data is also encouraged during peer review and at acceptance.

A more recent initiative to increase transparency is transparent peer review. Authors that have submitted after 1 December 2019 can opt into this process. Essentially, authors can choose to publish anonymous referee reports alongside author responses to those reviews upon publication. Potential referees are informed that authors are choosing this option when asked to review the manuscript. Uptake in Nature Communications, which introduced this option in 2016, has been high.

Correspondingly, Nature Immunology will be considering in the near future whether to offer referees the option of being named on the manuscripts they have peer reviewed, for better recognition.

Nature Immunology is also extremely cognizant of the importance of diversity in the immunology community. We recently highlighted this effort in a comment written by Akiko Iwasaki (https://doi.org/10.1038/ s41590-019-0470-6) and in a special issue on women in immunology (https://www. nature.com/collections/jdbaafddgb). In addition to raising these issues for our readers, we are actively trying to address diversity behind the scenes. We are aiming to address gender balance in our use of referees and commissioned content and to increase geographical diversity. In 2019, $58 \%$ of News and Views authors and $50 \%$ of
Review article authors were female. We have much to achieve going forward in improving diversity with other populations, and we still need to increase the percentage of female corresponding authors.

Nature Immunology has increased its variety of content over the last few years to accommodate the fast changes in the immunology field. To address the growing field of high-throughput research, we now publish Resource papers, which present a large data set (such as a comprehensive single-cell RNA sequencing data set or proteomic analysis) of broad utility, interest and significance to the community. A Letter format was introduced for papers that convey an important message to the community and thus should not necessarily be held up for publication by requiring more extensive mechanistic data, which could potentially hold the field back. We introduced Technical Reports for primary research data generated using a new technique that would likely be influential in the field. We also now welcome more human and translational research manuscripts. Finally, we have expanded our scope of commissioned articles to reflect awareness of the UN's Sustainable Development Goals; we now publish Comments and a new article type we call World View, which is a rather personalized point of view on matters pertaining to immunology, health and world issues.

While we recognize these are difficult times on so many levels, the editors have put together an anniversary issue (see https://www.nature.com/collections/ fddiddjdcj), which contains a series of Comments commissioned from some of our authors from the last 20 years that describe their landmark studies and how they drove immunology research forward in those particular areas. This issue features six previous Nature Immunology authors, and more Comments will be published in our Anniversary Series over the coming year. Finally, all of the editors wish to thank our authors and referees for their continued support of our journal. We look forward to at least another 20 years of stimulating and groundbreaking research that the immunology community will undoubtedly provide.

Published online: 23 June 2020 https://doi.org/10.1038/s41590-020-0729-y 